\title{
Results of Surgical Correction of Funnel-Shaped Deformation of the Chest Children
}

\section{IJCRR}

Section: Healthcare

Sci. Journal Impact

Factor: 6.1 (2018)

ICV: 90.90 (2018)

(c) (i) (5)

Copyright@IJCRR

\section{Bakhromjon Mirzakarimov', Jurakul Djumabaev², Karimjon Yulchiev ${ }^{3}$, Umidjon Mamajonov4, Doniyorbek Karimov5, Muzaffar Yuldashev6}

\begin{abstract}
'Ph.D., Docent, Head of Department of Pediatric Surgery, Andijan State Medical Institute, Andijan, Uzbekistan; ${ }^{2}$ Ph.D., Docent, Department of Pediatric Surgery, Andijan State Medical Institute, Andijan, Uzbekistan; ${ }^{3}$ Ph.D., Docent, Senior Teacher of the Department of Pediatric Surgery, Andijan State Medical Institute, Andijan, Uzbekistan; 'Senior Teacher of the Department of Pediatric Surgery, Andijan State Medical Institute, Andijan, Uzbekistan; 'Assistant, Department of Pediatric Surgery, Andijan State Medical lnstitute, Andijan, Uzbekistan; ${ }^{5}$ Assistant, Department of Pediatric Surgery, Andijan State Medical Institute, Andijan, Uzbekistan.
\end{abstract}

\section{ABSTRACT}

Introduction: Children have signs of chronic hypoxia, metabolic disorders. decreased appetite. Against this background, surgical treatment, which includes reconstruction of the chest, presents a certain risk. Based on these provisions, special attention was paid to the preoperative preparation of patients.

Objective: 1) Optimization of preoperative preparation and methods of surgical correction of funnel chest pectus excavatum( $\mathrm{PE})$; 2) Improvement of postoperative management of sick children with funnel chest.

Method: We operated 44 sick children in our hospital who were admitted to the surgical department with a diagnosis of PE. After preliminary examination, these patients underwent surgical correction of the chest according to Bairov and Ravich-Gross.

Results: In sick children aged 3-6 years after the Ravich-Gross operation, compared with the Bairov operation, the intensity of the pain syndrome was expressed as according to the VAS scale $(7.55 \pm 0.24$ versus $6.0 \pm 0.52$ points, $p<0.01)$ and on the VRS scale $(2.2 \pm 0.12$ versus $1.5 \pm 0.18$ points, $p<0.01)$. This plate provides more reliable fixation of the SCC than Marshev's tire, is convenient to use.

Conclusion: The methods of thoracoplasty with the use of external fixators are acceptable for children of the younger age group since these methods are safer in the long term than a metal plate inside the chest due to excessive motor activity of children. Good treatment outcomes are observed mainly in children operated on at the age of 3-6 years.

Key Words: Functional disorders, Funnel-shaped deformation, Marsheva's splint, Children, Conservative treatment

\section{INTRODUCTION}

As shown in the review of the literature with the age of the child, functional disorders become more pronounced, and against the background of frequent colds, tonsillitis, enlargement of the palatine tonsils, phenomena of chronic intoxication occur. ${ }^{9,11}$ Children have signs of chronic hypoxia, metabolic disorders decreased appetite. Against this background, surgical treatment, which includes reconstruction of the chest, presents a certain risk. Based on these provisions, special attention was paid to the preoperative preparation of patients. 2,3

\section{MATERIALS AND METHODS}

It seems to us that the surgical treatment of sick children should include two components: preoperative and operational. Preoperative (conservative) treatment is carried out for a long time (within 1-2 months, on an outpatient basis), including the sanitation of chronic foci of nasopharyngeal infection, general and extended chest massage with elements of breathing exercises. When patients were admitted to the hospital, clinical and laboratory, instrumental, and X-ray examinations were necessarily carried out and were also reexamined by narrow specialists (cardiologist, paediatrician, ENT). ${ }^{4,5}$

\section{Corresponding Author:}

Bakhromjon Mirzakarimov, Ph.D., Docent, Head of Department of Pediatric Surgery, Andijan State Medical Institute, Andijan, Uzbekistan; Email: jovohir99@mail.ru

ISSN: 2231-2196 (Print)

Received: 20.08 .2020
ISSN: 0975-5241 (Online)

Revised: 08.10 .2020
Accepted: 15.11 .2020 


\section{RESULTS AND DISCUSSION}

We operated 44 sick children in our hospital who were admitted to the surgical department with a diagnosis of PE. After preliminary examination, these patients underwent surgical correction of the chest according to Bairov and RavichGross, the distribution of which is presented in Table 1.

Table 1: Distribution of sick children by different
methods of surgical intervention
\begin{tabular}{|lcc}
\hline Methods of surgery & $3-6$ years & $7-15$ years \\
\hline According to Bairov & $6(23,1 \%)$ & - \\
According to Ravich-Gross & $20(76,9 \%)$ & $18(100 \%)$ \\
TOTAL & $26(100 \%)$ & $18(100 \%)$ \\
\hline
\end{tabular}

As can be seen from the table, the smallest number of children - 6 (23.1\%) underwent surgical intervention according to Bairov in the younger age group (3-6 years), since this method is characterized by low trauma and cosmetic properties. The Ravich-Gross operation was performed in 20 (76.9\%) children aged 3-6 years and in $18(100 \%)$ children aged 7-15 years, respectively. A characteristic feature of PEin older children is the progression of the disease, which is closely related to the growth and age of the child.

In this regard, the children of the older age group underwent the Ravich-Gross operation, taking into account the need for wide access to the SCC, additional incisions in the sternum, and ribs with the aim of effective correction and fixation of the SCC. For corrective thoracoplasty according to the Bairov and Ravich-Gross method, we used some modified versions of these operations, which will be indicated below (in the description of the operation).

These methods are based on resection of costal cartilage at the level of deformity, T-shaped sternotomy, traction for the body of the sternum using Marshev's splint. Both methods involve external fixation. The Marsheva splint is a lightweight, durable construction made of vinyl plastic and easy to sterilize. The tire is made taking into account the boundaries of the "funnel" before the operation (Figure 1).
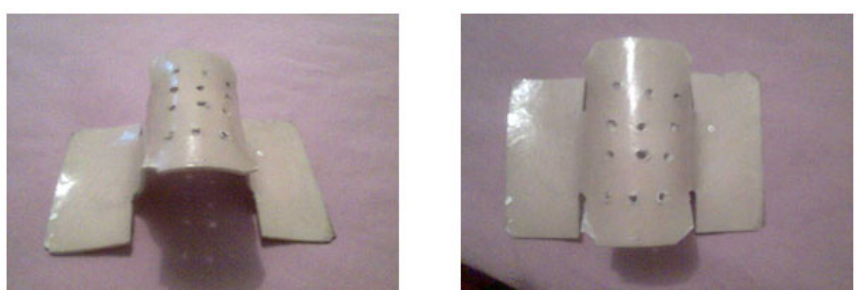

Figure 1: Marsheva splint (front and top view).

The immediate preparation of the patient for the operation began with premedication, the purpose of which is to potentiate the action of the main narcotic substance and conduct a neuro-vegetative blockade. Premedication was achieved by intramuscular injection in 30-40 minutes. before the operation, a solution of $0.1 \%$ atropine and $1 \%$ diphenhydramine in an age-specific dosage.

All operations were performed under total intravenous anaesthesia using artificial ventilation in a controlled breathing mode using Drager EV-801 and Blease Frontline 560 anaesthesia machines. We used a 5\% ketamine solution at a dose of $5-6 \mathrm{mg} / \mathrm{kg}$ as induction anaesthesia. which, to provide supportive drug therapy and infusions, a subclavian Seldinger catheter was installed in the modification of the staff of the Department of Pediatric Surgery with the course of anesthesiology at ASMI (Rational proposal No. 524 of June 14,2005 , by ASMI). For monoplegia, a $0.4 \%$ solution of pancuronium hydrobromide was used at a dose of $0.05 \mathrm{mg} /$ $\mathrm{kg}$. After preoxygenation of $100 \% \mathrm{O}_{2}$, the patients were intubated and transferred to apparatus breathing, $\mathrm{O}_{2}$ saturation was maintained at a level of $90-100 \%$, thereby creating a favourable condition for the surgeon to work on the chest wall. In children, we do not use gas-narcotic mixtures to maintain anaesthesia, given the functional disorders of the respiratory system, which is fraught with the development of complications in the respiratory system in the immediate postoperative period. To this end, a bolus of ketamine and an intravenous drip of propofol at a dose of $10 \mathrm{mg} / \mathrm{kg}$ was performed. As the main anaesthesia, $0.005 \%$ fentanyl was used at a dose of $1 \mathrm{ml} / \mathrm{kg}$, followed by the introduction of half the dose after 40 minutes. Intraoperative blood loss was replenished with a high-molecular-weight plasma substitute - Refortan 40 at a dose of $10 \mathrm{ml} / \mathrm{kg}$. After the completion of the operation and the appearance of spontaneous breathing, all patients were extubated on the operating table followed by $\mathrm{O}_{2}$ insufflation and transferred to the post-anaesthetic ward of the intensive care unit under the supervision of an anesthesiologist.

\section{Operation technique according to Bairov}

According to this method, 8 children aged 3-6 years were operated with some modifications, which are as follows:

The position of the patient on the back, a flat roller was placed under the shoulder blades. At the edge of the impression, 4 small longitudinal skin incisions $(3-4 \mathrm{~cm})$ were made in such a way that each wound could be processed above and below the rib. Slightly shifting the skin wound to the apex of the rib curvature and stupidly stratifying the muscles above it, trying not to damage the pleura and blood vessels, the necessary segment of the rib was resected, which was calculated on the contourogram, without affecting the growth zone (the place of transition of the bone part into the cartilaginous one) and immediately the ends of the resected ribs are sutured lavsan threads. After that $(4-5 \mathrm{~cm})$, an incision was made in the skin and subcutaneous tissue over the base of the xiphoid process, which, unlike the traditional method, was not cut 
off from the sternum. At the same time, the retrosternal ligament was revealed - a dense cord running from the posterior surface of the xiphoid process to the diaphragm. After mobilization of the retrosternal ligament, a Z-shaped excision of the latter was performed, with the calculation of the required distance (determined on the contourogram), which is equal to half this value.

Then, bluntly (with a finger) peeled off from the inner surface of the sternum adjacent sheets of the parietal pleura and pericardium. After that, we proceed to mobilize the deep section of the sternum. Above the beginning of the curvature of the sternum, a T-shaped sternotomy was performed with a thin chisel or scalpel (under the control of a finger inserted behind the sternum), and the inner plate was fractured by applying pressure from the inside. After sternotomy, the mobility of the sternocostal was checked, if tensions were noted, additional incisions were made in the tension area. Under the control of a finger, the mobilized sternum was percutaneously held with a fishing line for subsequent stretching and fixation. The previously excised retrosternal ligament was sutured end-to-end (Figure 2).
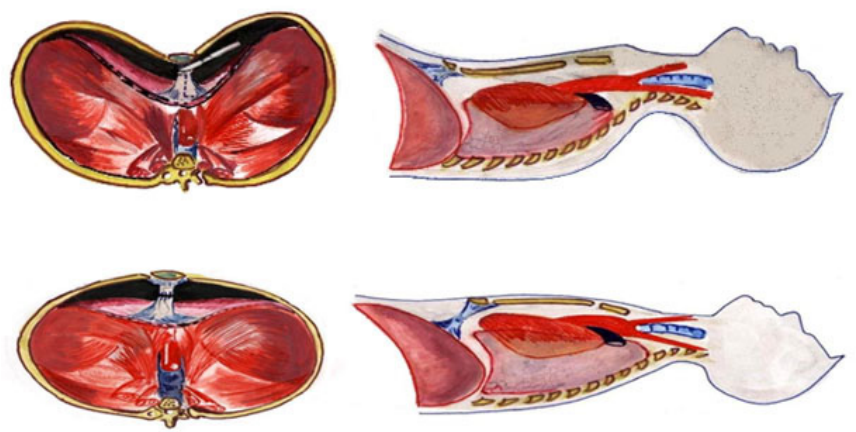

Figure 2: Mobilization of the Sterno-phrenic ligament, Zshaped excision, end-to-end stitching.

Then the wounds were sutured in layers, the withdrawn traction threads were passed through the corresponding holes of Marshev's special vinyl plastic splint and tied over gauze balls with a tension sufficient to hold the aligned part of the sternum and ribs. The splint was placed on a foam or rubber sponge, created for constant stretching and fixation, the structure was closed with a light gauze bandage on a cleola. The average duration of the operation ranged from 1 hour to 1 hour. 20 minutes. Immobilization was carried out depending on age for 20-30 days, followed by replacement of Marshev's splint with a plate.

\section{Operation technique according to Rawicz-Gross}

A transverse wave-like (submammary) incision was made for $15-20 \mathrm{~cm}$. The tissues were dissected in layers and the skin and subcutaneous tissue were mobilized on both sides of the incision, within the limits of deformation after hydrau- lic dissection by the introduction of $0.25 \%$ Novocain. The length of the resection was established before the operation by contouring calculation. Immediately after resection, the edges of the ribs were connected with separate lavsan sutures. Then, in the region of the xiphoid process, a semilunar incision was made, mobilizing the retrosternal ligament, and a Z-shaped excision of the latter was performed. The pleura and pericardium were mobilized from the inner surface of the deformed sternum. A T-shaped sternotomy was performed with excision of the wedge at the transverse intersection of the anterior plate (we break the posterior plate) under the control of a finger. Through the body of the sternum in the middle part, 2 threads (fishing line) were passed for subsequent stretching. Z-shaped excised retrosternal ligament was sutured end to end with a nylon thread. The defect over the ligament was sutured with nylon thread. The superimposed traction threads were passed out through separate skin punctures. The wound surface was treated with an antibiotic solution. The subcutaneous tissue and skin were sutured. The withdrawn traction threads were fixed to Marshev's splint after achieving the visual correction of the breast. ${ }^{6,7}$

The two modified methods of surgical correction of funnel chest used by us are effective in cosmetic terms and in terms of eliminating cardiorespiratory disorders, are less traumatic with a low risk of intraoperative complications in the form of damage to the pleura and pericardium. To ensure less trauma in the intra-, postoperative period when performing these operations, we have optimized and modified the following points:

Firstly, when mobilizing the sternum during the operations of Bairov and Ravich-Gross, we did not resort to exfoliation of the parietal pleura and pericardium from the inner surface of the deformed ribs, but limit ourselves only to exfoliating them from the inner surface of the sternum, which prevents damage to the pleura and the occurrence of pneumothorax during the operation eliminates the need for X-ray control.

Secondly, in contrast to traditional methods, with both methods, we performed a complete resection of curved ribs along the edge of the "funnel". In our opinion, subperichondral resection may lead to further growth at the junction of the resected ribs (exostosis), caused by the infringement of the perichondrium in the attachment area. Also, children with PE have a hereditary predisposition to exostosis associated with dysplasia of connective tissue elements.

Thirdly, we immediately sew the ends of the resected ribs with lavsan threads. This stage of the operation, in our opinion, has a fundamental character, because, after the mobilization of the SCC, some displacement of the resected ribs is noted, which can lead to their erroneous stitching.

Fourthly, unlike the traditional method, the xiphoid process together with the retrosternal ligament was not cut off from 
the sternum. We believe that cutting off the xiphoid process together with the retrosternal ligament can lead to hypercorrection in the long-term postoperative periods; also, the retrosternal ligament helps to keep the SCC in a fixed position from the inside when pulling on Marshev's bus, which ensures the prevention of overcorrection..$^{9,10}$

Fifth, after mobilization of the retrosternal ligament, a Zshaped excision of the latter was performed, with the calculation of the required distance (determined on the contourogram), which is equal to half of this value, which was sutured end to end, which prevents hypercorrection and recurrence of deformity in the postoperative period. The average duration of the operation ranged from 1 hour 20 to 1 hour. 40 minutes. ${ }^{7,8}$

Numerous studies have shown ${ }^{2,8}$ that insufficient postoperative pain relief leads to negative changes in the emotional state of the child and increases the number of complications. So, hypoventilation in children after thoracoplasty is associated with the development of pain. Pain syndrome is an integral part of the postoperative illness and is often the root cause of a variety of disorders and deviations in the condition of patients. Therefore, an effective fight against pain in the postoperative period should be considered not only as a desire to alleviate the patient's physical suffering, improve his psycho-emotional state, but also as a prevention of hemodynamic, gas exchange, and metabolic disorders. Assessment of the pain factor in the postoperative period is complicated to a large extent by the fact that for a certain time the factor of post-anaesthetic depression acts, the degree of blood loss, disturbance of CBS, temperature balance, and several other factors matters. In this regard, in our clinic, to assess the intensity of acute pain, the Visual Analog Scale (VAS) and the four-digit categorical verbal scale (Verbal Rating Scale, VRS) are used, which are equally sensitive for determining acute postoperative pain.

At the same time, it was found that in sick children aged 3-6 years after the Ravich-Gross operation, compared with the Bairov operation, the intensity of the pain syndrome was expressed as according to the VAS scale $(7.55 \pm 0.24$ versus $6.0 \pm 0.52$ points, $p<0.01)$ and on the VRS scale $(2.2$ \pm 0.12 versus $1.5 \pm 0.18$ points, $p<0.01$ ). Pain syndrome in sick children who underwent surgery at the age of 7-15 years was characterized by a lower severity compared with children aged 3-6 years - VAS $(6.15 \pm 0.31$ versus $7.55 \pm$ $0.24, \mathrm{p}<0,01)$ and VRS $(2.1 \pm 0.10$ versus $2.2 \pm 0.72$ points, $\mathrm{p}>0.05$ ). Consequently, the intensity of the pain syndrome is more pronounced after the Ravich-Gross operation than the Bairov operation, as well as at the age of 3-6 years due to their high emotional lability, pain sensitivity.

It is known that the use of traditional narcotic analgesics (promo, fentanyl, morphine, etc.) for postoperative analgesia in children, especially the younger age group, leads to several side effects and undesirable reactions of these drugs such as sedation, respiratory depression and dyspeptic disorders (nausea, vomiting).

Based on the above provisions, for postoperative analgesia, we used the drug Ketonal (Lek Company, Slovenia) at a dose of $50 \mathrm{mg}$ per administration. The indications for use were strong and moderate pain syndrome. The severity of the pain syndrome determined the frequency of administration of ketonal, but no more than three times per day. When developing a plan for pharmacotherapy for pain, we proceeded from two key principles: 1) The principle of an individualized approach: the analgesic efficacy of drugs can vary widely in the same patient. In this regard, the dose, the route of administration, as well as the dosage form were determined strictly individually, taking into account the intensity of pain and based on regular monitoring; 2) The principle of timeliness of administration: The interval between injections of the drug should be determined by the severity of pain and the pharmacokinetic characteristics of the drug and its dosage form. Doses should be given regularly to prevent pain, not relieve it after it occurs.

Comparing the quality and analgesic efficacy of pain relief by traditional narcotic analgesics with a non-steroidal nonnarcotic analgesic - ketonal, as well as the severity of their side effects, it was found that in the group of children who received ketonal as postoperative analgesia, the effectiveness of analgesia was recognized as good. There were no hemorrhagic disorders characteristic of non-steroidal antiinflammatory analgesics. There were also no characteristic dyspeptic disorders (nausea and vomiting) and - sedation, respiratory depression.

Thus, ketonal, combining sufficient analgesic activity and the absence of disadvantages inherent in narcotic analgesics, provides adequate postoperative pain relief in children operated on for chest deformities.

On the second day of the postoperative period, management and observation were carried out in the department of surgery. The process of postoperative management of sick children is conditionally divided by us into two stages: the early (inpatient) stage - the period of hospital stay (8-9 days), the late (outpatient) stage - the observation of patients after discharge home until the removal of internal and external fixators. In the stationary stage of the postoperative period, the management of sick children before discharge was as follows:

Day 1: Toilet of the wound, treatment of sutures, traction threads with $3 \%$ tincture of iodine and alcohol after operations of Bairov, Ravich-Gross, allow a sitting position, continue antibacterial therapy, and anaesthesia.

Day 2: Toilet wounds, treatment of sutures, traction threads, allow walking, a continuation of antibiotic therapy, and pain relief. 
Day 3: Toilet wounds, treatment of stitches, traction threads, anaesthesia according to indications.

Day 4,5,6: Toilet wounds, treatment of sutures, traction threads, antibacterial therapy, pain relief according to indications.

Day 7: Wound toilet, treatment of stitches, traction threads, removal of stitches with alternation. Cancellation of antibiotics.

Day 8: Wound toilet, treatment of stitches, traction threads, removal of stitches. Extract.

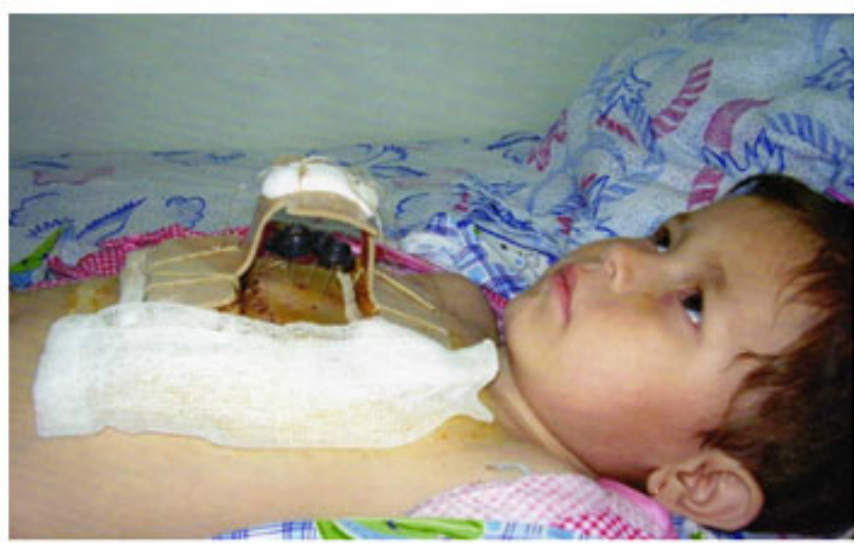

Figure 3: General view of the patient with an external fixator (Marshev's splint).

Re-examination of the patient after operations according to the Bairov and Ravich-Gross method was carried out ten days later, the condition of the wound, external fixator, and GRC in a fixed position was assessed. In the presence of a weakening of the traction threads, stretching was carried out, and in case of a tendency to overcorrection, weakening of the traction threads, wound treatment, the imposition of an aseptic bandage (Figure 3 ).

According to domestic and foreign researchers, Marshev's splint allows us to reliably hold the sternocostal complex after thoracoplasty, and early activation of the patient and reduces hospital stay. Immobilization was carried out depending on age and was 20-30 days. Ultrasound studies of costal cartilage in the area of resection show that the process of cartilage regeneration is accompanied by the development and involution of blood vessels in the resection area. ${ }^{8}$ From 2-3 months. after thoracoplasty, the involution of blood vessels begins, which ends by 4-6 months. These scientific prerequisites provide a basis for the fact that the timing of the immobilization of the SCC using external traction is insufficient. Nevertheless, thoracoplasty with the use of external traction of the SCC can be accompanied by the eruption and detachment of traction devices and infection of the chest wall tissues along the threads and strings. ${ }^{12,13}$ Moreover, prolonged use of the external splint leads to discomfort, displacement of the retainer when changing the position of the body due to its significant size, and prevents the patient from returning to normal life as soon as possible. Considering the above, for long-term and sufficient immobilization of the GRC in terms of time, we use a flat, plastic arcuate plate, prepared to depend on the age and size of the chest (rational proposal No. 2117 dated 02.06.2008 by ASMI) (Figure 4). The plate is installed from 3 to 4 months, under supervision every 20 days.
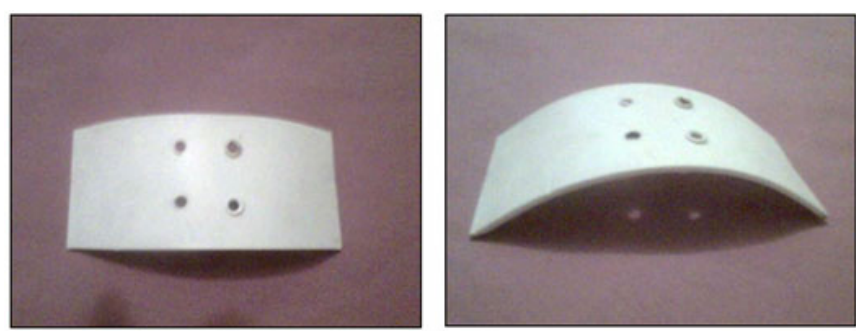

Figure 4: Arcuate plate (top and side view).

This plate provides more reliable fixation of the SCC than Marshev's tire, is convenient to use, does not restrict the movement of the child, does not require additional help from parents when changing the position of the body, also, such undesirable phenomena as displacement of the plate, eruption of threads, detachment of traction devices, wound infection along the threads, ${ }^{13,14}$ as well as is convenient for monitoring the state of the sternocostal complex in a corrected position (Figure 5).
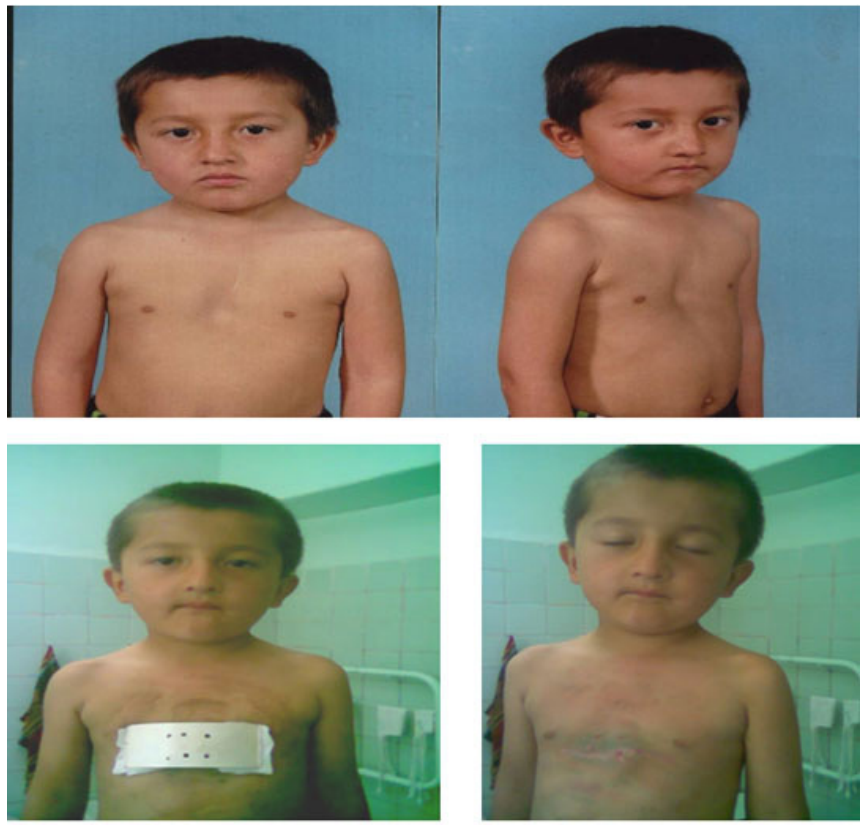

Figure 5: View of the patient after 3 months with the plate and after removal. 
In the immediate postoperative period, complications were noted, such as hemodynamic disturbances in $32.1 \%$ ( $p \varphi$ $<0.001)$, secondary healing in $21.1 \%(p \varphi<0.001)$ cases, as well as recurrence of deformity in $25.0 \%(p \varphi<0.001)$, hypercorrection of the sternocostal complex in $10.7 \%$ (p $\varphi$ $<0.003$ ) and keloid scarring of the wound surface in $14.3 \%$ $(p \varphi>0.05)$ of sick children in the late postoperative period.

The above complications were observed much less frequently during treatment and management according to our method. They were observed in the form of pneumothorax in $2.63 \%$ ( $p \varphi<0.013)$, intraoperative bleeding in $5.26 \%$ (p $\varphi$ $<0.004)$, prolonged surgery in $5.26 \%$ (p $\varphi<0.001)$, hemodynamic disorders in $2.63 \%(\mathrm{p} \varphi<0.001)$, recurrence of deformity in $2.63 \%(\mathrm{p} \varphi<0.001)$ and formation of keloid scars in $5.26 \%(p \varphi>0.05)$, respectively.

Consequently, during the traditional version of the RavichGross operation before using our version of the operation, intra- and postoperative complications were observed in $29.5 \%$ of the operated children, and after using the proposed optimized version of the Ravich-Gross operation, this figure decreased to $3.95 \%(p \varphi<0.001)$, i.e., by more than 7 times.

\section{CONCLUSION}

The methods of thoracoplasty with the use of external fixators are acceptable for children of the younger age group since these methods are safer in the long term than a metal plate inside the chest due to excessive motor activity of children. Good treatment outcomes are observed mainly in children operated on at the age of 3-6 years. This age is considered optimal for the surgical correction of this malformation. Z-shaped lengthening of the retrosternal ligament makes it possible to avoid the recurrence of deformity in the long term after the operation. The use of a modified plate makes it possible to extend the sternum for a longer period, i.e., a more durable fusion of the resected ends of the ribs.

\section{ACKNOWLEDGMENT}

Authors acknowledge the immense help received from the scholars whose articles are cited and included in references to this manuscript. The authors are also grateful to authors / editors / publishers of all those articles, journals, and books from which the literature for this article has been reviewed and discussed.

Conflict of interest: Authors declare that they have no conflict of interest.

\section{Financial support: None}

\section{REFERENCES}

1. Berker AN, Yalçın MS. Cerebral palsy: orthopaedic aspects and rehabilitation. Pediatr Clin North Am 2008;55(5):1209-25.

2. Mutch L, Alberman E, Hagberg B, Kodama K, Perat MV. Cerebral palsy epidemiology: where are we now and where are we going? Dev Med Child Neurol 1992;34(6):547-51.

3. Sankar C, Mundkur N. Cerebral palsy-definition, classification, aetiology and early diagnosis. Indian J Pediatr 2005;72(10):8658.

4. Kilincaslan A, Mukaddes NM. Pervasive developmental disorders in individuals with cerebral palsy. Dev Med Child Neurol 2009;51(4):289-94.

5. Singhi PD, Ray M, Suri G. Clinical spectrum of cerebral palsy in North India - an analysis of 1000 cases. J Trop Paediatr 2002;48(3):162-6.

6. Rosen MG, Dickinson JC. The incidence of cerebral palsy. Am J Obstet Gynaecol 1992;167(2):417-23.

7. Winter S, Autry A, Boyle C, Yeargin-Allsopp M. Trends in the prevalence of cerebral palsy in a population-based study. Paediatrics 2002;110(6):1220-5.

8. Hagberg B, Hagberg G, Zetterström R. Decreasing perinatal mortality-increase in cerebral palsy morbidity? Acta Paediatrica 1989;78(5):664-70.

9. Yoon BH, Jun JK, Romero R, Park KH, Gomez R, Choi JH, Kim IO. Amniotic fluid inflammatory cytokines (interleukin-6, interleukin-1 $\beta$, and tumour necrosis factor- $\alpha$ ), neonatal brain white matter lesions, and cerebral palsy. Am J Obstet Gynaecol 1997 Jul 1;177(1):19-26.

10. Wu YW, Colford Jr JM. Chorioamnionitis as a risk factor for cerebral palsy: a meta-analysis. JAMA 2000;284(11):1417-24.

11. Mallick S. Study On The Clinical Profile Of Patients With Cerebral Palsy (Doctoral dissertation) 2011; 52(172):127-47.

12. Garg SA. Chakravarti R. Singh NR, Masthi RC, Goyal GR. Jammy E. Dengue serotype-specific Seroprevalence among 5to 10-Year-Old Children in India: A Community-Based CrossSectional Study. Int J Infect Dis 2017; 54:25-30.

13. Puri S, Fernandez SA, Puranik D, Anand A, Gaidhane Z, Quazi S, et al. Policy Content and Stakeholder Network Analysis for Infant and Young Child Feeding in India. BMC Public Health. 2017; 17:217-21

14. Taksande A, Meshram R, Lohakare A. A Rare Presentation of Isolated Oculomotor Nerve Palsy Due to Multiple Sclerosis in a Child. Int J Pediatr 2017; 5(8): 5525-29. 